Int. J. Odontostomat., 11(1):53-60, 2017.

\title{
Correlación entre el Comportamiento Térmico y Composición de Sistemas Adhesivos
}

\author{
Correlation between Thermal Behavior and Composition of Adhesives Systems
}

\author{
Lilian Nass Kunstmann ${ }^{1}$ \& Agata Rita Carpentieri ${ }^{2}$
}

\begin{abstract}
NASS, K. L. \& CARPENTIERI, A. R. Correlación entre el comportamiento térmico y composición de sistemas adhesivos. Int. J. Odontostomat., 11(1):53-60, 2017.

RESUMEN: Se realizó un estudio in vitro donde se evaluaron once sistemas adhesivos, para establecer si las condiciones de trabajo clínico favorecen su eficiencia. Se plantea que los sistemas adhesivos autoacondicionantes presentan menor estabilidad térmica que los convencionales. Determinar el comportamiento térmico de once sistemas adhesivos sometidos a diferentes rangos de temperatura en ambientes con $100 \%$ de humedad, agua acidulada, humedad ambiental, saliva artificial y la correlación con su composición. Se utilizaron adhesivos autograbantes y convencionales, se prepararon muestras en cápsulas de aluminio pequeñas y se fotopolimerizaron con una lámpara LED. Se les realizó el estudio de espectroscopía infrarrojo con transformada de Fourier (FT-IR), asignando las bandas de absorción de los grupos funcionales orgánicos de cada compuesto, correspondientes a los grupos químicos que poseen. Posteriormente fueron sometidas a examen termogravimétrico entre temperatura ambiente y $500^{\circ} \mathrm{C}$ para establecer un patrón de comportamiento térmico en ambiente inerte y luego de permanecer en agua acidulada, $100 \%$ humedad, humedad ambiental y saliva artificial. Los termogramas informaron las temperaturas de descomposición y los porcentajes de pérdida de masa. Se correlacionaron los resultados del estudio de FT-IR infrarrojo con el comportamiento térmico de los sistemas adhesivos. Se observó pérdida de masa, eficiencia de la polimerización y cantidad de masa residual. Se observaron dinámicas de degradación diferentes por el diseño de las curvas y por los cambios en la línea base. Conclusión: Los sistemas adhesivos de ambos grupos analizados, presentaron elevado grado de polimerización. Sin embargo, el efecto producido por las condiciones a las cuales fueron sometidos, depende del tipo de adhesivo, siendo que, los adhesivos convencionales, a diferencia de los autoacondicionantes, se degradan en menor extensión, reflejado por su mayor estabilidad térmica.
\end{abstract}

PALABRAS CLAVE: sistemas adhesivos, estabilidad térmica, degradación hidrolítica.

\section{INTRODUCCIÓN}

Los sistemas adhesivos poliméricos pertenecen a la familia de las resinas compuestas, y de acuerdo a la clasificación según su viscosidad, son fluidos (Abate, 2013). Son los protagonistas de la técnica adhesiva, paso previo de vital importancia en la aplicación de una restauración de resina compuesta o en el procedimiento de cementación de una restauración.

A pesar de sus ventajas, las resinas compuestas presentan significativas deficiencias en cuanto a su desempeño, sobre todo lo relacionado con la contracción de polimerización y al estrés que ésta produce en la interface diente-restauración. Actualmente, las mejoras en las formulaciones, el desarrollo de nuevas técnicas de colocación y la optimización de sus propiedades físicas y mecánicas, han hecho la restauración de resina compuesta más segura y predecible (Rodríguez \& Pereira, 2007).

Una buena unión al esmalte dentario es muy importante para preservar la interface diente restauración en términos de permanencia de la restauración en la cavidad bucal en condiciones óptimas, indicándose acondicionar siempre el esmalte con ácido fosfórico para garantizar la adhesión (De Munck et al., 2005). Artículos científicos demuestran que se consigue una excelente adhesión in vitro con sistemas adhesivos poliméricos autoacondicionantes de dos

\footnotetext{
${ }^{1}$ Facultad de Odontología, Universidad de Concepción, Concepción, Chile.

${ }^{2}$ Facultad de Odontología, Universidad Nacional de Córdoba, Córdoba, Argentina.
} 
pasos con el esmalte biselado, no intacto, siendo apenas diferente a nivel de la integridad marginal in vivo (Perdigão et al., 2005; Eminkahyagil et al., 2005) y mejor cuando se realiza el grabado con ácido fosfórico previo en el esmalte (Fuks et al., 2002; Hannig et al., 2004; Peutzfeldt \& Nielsen, 2004).

Los sistemas adhesivos poliméricos se pueden clasificar de diferentes maneras para entender su evolución. Sin embargo parece más sensato clasificarlos según su estrategia adhesiva en los que requieren las acciones de grabar y lavar, que pueden ser de tres 0 dos pasos, y los autoacondicionantes o autograbantes, que son de dos y un paso (Perdigão et al.). El sistema adhesivo convencional de 3 pasos, de grabado total con primer basado en etanol, presenta un comportamiento clínico y de laboratorio superior, siendo el «Gold estándar» en la adhesión dentina - resina (De Munck et al., 2005).

Los sistemas adhesivos convencionales de 3 pasos y los autograbantes de 2 pasos, dan resultados clínicos confiables. Los sistemas que buscan una técnica de aplicación más simplificada, presentan mayor sensibilidad a la técnica y menor rendimiento clínico, siendo los sistemas adhesivos de 2 pasos con grabado total, los más susceptibles a la degradación (Peumans et al., 2005). Una banda de resina compuesta adherida a esmalte, rodeando a la resina compuesta adherida a dentina, protege efectivamente la degradación de la capa adhesiva y la restauración. La mayor implicancia clínica de este fenómeno se observa en el margen gingival de restauraciones clase II, donde la presencia de esmalte es lo ideal para el éxito clínico a largo plazo (Gamborgi et al., 2007), por lo que no se recomienda restaurar una clase II en forma directa si no existe esmalte cervical, siendo una restauración indirecta una mejor opción para obtener mejor ajuste cervical y menor contracción de polimerización por volumen de resina, ya que sólo se utiliza en forma directa la resina de fijación.

En relación al comportamiento clínico de sistemas adhesivos con y sin relleno, se concluye que a pesar de que los adhesivos con relleno mejoran levemente la durabilidad, esta no es significativa (Ritter et al., 2009). Los patrones de degradación dentro de la capa híbrida están representados por pérdida de resina de los espacios interfibrilares y desorganización de las fibrillas de colágeno, debido a la hidrólisis de la resina y/o el colágeno (Breschi et al., 2008). Los sistemas adhesivos autograbantes de un paso presentan mayor absorción de agua. La resina hidrófila HEMA aumenta la absorción de agua y disminuye la resistencia a la tracción presentando dominios hidrófilos con solubilidad del sistema adhesivo, esto relacionado a su hidrofilicidad. Al utilizar sistemas adhesivos sin HEMA, se observa nanofiltración en racimos de agua en la capa adhesiva, debido a la fase de separación de los componentes hidrófugos y el agua residual en la superficie de la dentina (Takahashi et al., 2011).

Por el momento se puede afirmar que las resinas a base de metacrilato y sus adhesivos son muy sensibles a la técnica. Además, si el sistema adhesivo no impregna bien la estructura dentaria, la restauración fallará en poco tiempo, debido a la percolación marginal, teniendo como consecuencia sensibilidad postoperatoria, pigmentación de la zona afectada, caries recidivante y pérdida de la obturación, como un proceso cronológico predecible (Tay et al., 1996; Sano et al., 1999; Hashimoto et al., 2000; Donassolo et al., 2005; Spencer et al., 2006; Kostoryz et al., 2009).

En el presente trabajo de investigación, se intenta establecer el efecto de cuatro ambientes: agua, $\mathrm{pH} 3$, humedad ambiental y saliva artificial en once sistemas adhesivos poliméricos, autograbantes y convencionales, para establecer la correlación entre la pérdida de masa y su composición. Se realizó un estudio exhaustivo de sistemas adhesivos poliméricos existentes en el mercado para destacar aquellos que presentan mejor comportamiento, entendiendo que ello contribuye al éxito clínico. Un adhesivo muy hidrófilo en contacto con humedad en boca se degrada y pierde masa, favoreciendo la necesidad de reemplazar restauraciones estéticas insatisfactorias a corto o mediano plazo (Leinfelder, 2000).

\section{MATERIAL Y MÉTODO}

Los cuatro adhesivos autograbantes utilizados fueron: Adper Silorano (3M Espe), Futurabond DC (VOCO), Go! (SDI) y AdheSe (Vivadent), entre los cinco adhesivos monofrasco que se analizaron estaban: Prime \& Bond NT (Dentsply), Adper Single Bond 2 (3M Espe), Solobond M (VOCO), One Coat Bond (Coltene), Optibond Solo Plus (Kerr) y XP Bond (Dentsply).

Con cada sistema adhesivo se prepararon 40 muestras, conformando 11 grupos con 40 muestras cada uno. Para cada grupo, se colocaron $10 \mathrm{mg}$ aproximadamente de cada uno de los adhesivos, en cápsu- 
las de aluminio pequeñas. El proceso de fotopolimerización se realizó con una lámpara LED Radii Cal de SDI de acuerdo a las indicaciones de los fabricantes, previamente testeada con un radiómetro digital, el cual indicó $2.100 \mathrm{mw} / \mathrm{cm}^{2}$ para que la lámpara sea utilizada en cada muestra siguiendo los protocolos clínicos.

Para estandarizar la distancia de fotopolimerización, entre el espécimen y la lámpara LED, se posicionó un protector ocular que se emplea en la punta activa de la boquilla de la lámpara. Este protector se interpuso entre el protector habitual de la lámpara y la muestra. La altura del protector es de 5,4 $\mathrm{mm}$. La altura de la cápsula de aluminio es de 1,6 mm estando la superficie de la boquilla de la lámpara a 4 $\mathrm{mm}$ de la superficie del adhesivo aproximadamente. Se obtuvieron de esta forma un total de 440 muestras.

Preparación de pastilla para análisis infrarrojo: De los 11 grupos, se procedió a elegir en forma aleatoria 2 muestras para comprobar que poseen la misma composición y verificar variables de polimerización, a las que se les fraccionó un trozo, obteniéndose un total de 22 muestras. Luego se molieron en un mortero de ágata, homogenizando con $100 \mathrm{mg}$ de bromuro de potasio $(\mathrm{KBr})$, como soporte de las muestras para análisis por espectroscopía infrarrojo FT-IR. Se llevó la muestra molida a una prensa hidráulica, se mantuvo con una carga de 5 toneladas métricas, con 10.000 libras de presión por 1 minuto para la confección de una pastilla. La pastilla preparada se llevó al equipo FT-IR Nicolet modelo NEXUS, para su estudio con láser Neón Helio y obtención de los gráficos.

Se estudió la composición y caracterización cualitativa, asignando a las bandas de absorción de los espectros, los grupos químicos que ellos poseen. Se señalaron los grupos funcionales orgánicos que se presentaron en forma más relevante, para saber con cierta certeza qué tipo de monómeros dieron origen al polímero.

Estudio por espectroscopía infrarrojo FT-IR: La técnica instrumental utilizada para establecer la composición e identificación de grupos funcionales, fue la espectroscopía infrarroja con transformada de Fourier. Adicionalmente, esta técnica se utilizó para evaluar los cambios de grupos funcionales inducidas por la polimerización y que permiten establecer el grado de conversión. Del espectrofotómetro FT-IR se obtiene los espectros de infrarrojo utilizando el método por transformaciones de Fourier, para la determinación de la composición del polímero (Rouessac \& Rouessac, 2000).

Estudio por termogravimetría (TGA): El resto de las muestras se mantuvieron a ambientes asociados a su uso clínico, entre ellos; $\mathrm{pH} 3$, degradación hidrolítica, degradación con saliva artificial, y como control, se dejaron muestras en condiciones ambientales. Se mantuvieron por una semana a $37^{\circ} \mathrm{C}$ y luego fueron sometidas a análisis Análisis Térmico Gravimétrico (TGA) para establecer un patrón de comportamiento térmico en un rango comprendido entre temperatura ambiente y $500^{\circ} \mathrm{C}$. Los termogramas informaron las temperaturas de descomposición y los porcentajes de pérdida de masa de los sistemas adhesivos. Posteriormente, se correlacionaron los resultados del estudio por análisis infrarrojo con el comportamiento térmico, para establecer una dinámica de correlación entre pérdida de masa y su composición.

Todas las muestras fueron analizadas usando TGA. Esta técnica permite establecer la estabilidad térmica relacionando esta variable con la eficiencia de la polimerización y efecto de las condiciones a que fueron sometidas las muestras. De esta manera, se estableció los efectos degradativos inducidos por los diversos ambientes a que fueron sometidos los sistemas adhesivos. Esta característica se asocia a los diferentes patrones de las curvas de pérdida de masa, que presentan los diferentes adhesivos y se asocian a la degradación inducida por los ambientes.

Para el análisis TGA, se depositó cada sistema adhesivo, previamente molido en un mortero de ágata, en tubos capilares de $5 \mathrm{ml}$ y se prepararon los grupos. El medio con $100 \%$ de humedad se consiguió inundando de agua la muestra de adhesivo ubicada en el tubo capilar. En el ambiente de agua acidulada se adicionaron $5 \mathrm{ml}$ de solución buffer a pH 3 y el grupo con saliva artificial se preparó agregando $5 \mathrm{ml}$ de ella a los tubos capilares. Todos los tubos capilares fueron tapados con un trozo de parafilm, mientras que las muestras del grupo en humedad relativa se mantuvieron sin tapar.

Se distribuyeron los sistemas adhesivos en los diferentes ambientes y se mantuvieron en una estufa por una semana a $37^{\circ} \mathrm{C}$. Posteriormente, se colocaron en una estufa al vacío para eliminar la porción húmeda, a través del siguiente protocolo: inicialmente se mantuvieron 1 hora a $80^{\circ} \mathrm{C}$, posteriormente, 1 hora a $70^{\circ} \mathrm{C}$, a una presión atmosférica de 0,1 pulgada de 
Hg. Luego se colocaron las muestras en un desecador con sílica gel para preservarlas de la humedad en el traslado al laboratorio, donde se realizaron los análisis TGA.

Para el estudio de la estabilidad térmica de los sistemas adhesivo en cada uno de los cuatro ambientes, se sometieron a temperaturas desde los $37^{\circ} \mathrm{C}$ a $500{ }^{\circ} \mathrm{C}$ y luego se enfriaron hasta llegar a la temperatura ambiente en atmósfera inerte (nitrógeno gaseoso). El proceso para cada muestra duró una hora aproximadamente, dependiendo de la velocidad de calentamiento. Se determinó el grado de pérdida de masa por descomposición del material al ser sometido a calentamiento y enfriamiento. Los resultados dieron una gráfica de descomposición propia de cada material, entregando información sobre temperaturas de descomposición, porcentajes de pérdida de masa y carga residual. Los termogramas o curvas de descomposición térmica informaron las temperaturas de descomposición y los porcentajes de pérdida de masa.

Instrumentos de medición: Estudio Infrarrojo: equipo Infrarrojo con Transformación de Fourier FTIR Nicolet modelo NEXUS. Estudio Térmico: se realizó mediante análisis termogravimétrico (TGA). Las especificaciones del equipo son: Analizador Termogravimétrico Thermo Microbalance TG 209 F1 IRIS acoplado a Espectrómetro de Masa (QMS). Marca NETZSCH. En atmósfera inerte (nitrógeno gaseoso).

Para el análisis de la estabilidad térmica, se registraron los datos mediante el análisis de los termogramas, realizando una comparación simultánea de las curvas. Además se confeccionó una base de datos común, incluyendo todos los sistemas adhesivos en los distintos medios o ambientes. Esta base de datos fue exportada al programa InfoStat 2013, el que permitió visualizar los gráficos de dispersión de cada uno los sistemas adhesivos en todos los ambientes estudiados.

\section{RESULTADOS}

Se analizaron cualitativamente los datos arrojados en los gráficos de los infrarrojos. Los resultados obtenidos reflejaron la composición de sistemas adhesivos poliméricos en forma cualitativa, de acuerdo a la información entregada por los fabricantes en los instructivos.

En las Tablas I y II se indica los monómeros que contienen los sistemas adhesivos convencionales y autoacondicionantes, sus marcas comerciales y sus respectivos solventes. En los gráficos TGA, se observan dos curvas: las curvas de $\%$ de pérdida de masa versus temperatura ( $\%$ pérdida de masa vs $\mathrm{T}^{\circ}$ ), que corresponden a las curvas integrales, y las curvas de $\%$ de pérdida de masa dividido por temperatura vs temperatura, correspondientes a las curvas diferenciales o primeras derivadas (Fig. 1). A partir del análisis de los termogramas de los once sistemas adhesivos poliméricos, en los cuatro ambientes a los cuales fueron expuestos, se obtuvieron los gráficos con la curva integral y la curva correspondiente a la primera derivada o diferencial termogravimetría (DTG), entregados por el software Proteus NETZSCH del equipo TGA.

La primera derivada corresponde a la temperatura donde ocurre la máxima velocidad de pérdida de masa (TD) y muestra con mayor detalle los procesos de descomposición. Los sistemas adhesivos de grabado independiente o sistemas adhesivos convencionales resultaron ser los sistemas adhesivos con mejor estabilidad térmica en todos los ambientes estudiados, en donde se puede observar que las temperaturas a las que comienza la degradación en todos los casos, es una temperatura superior a la que presentan los sistemas adhesivos autoacondicionantes (Fig. 2). De acuerdo a la tendencia de los resultados, se confirma la hipótesis planteada en esta investigación, es decir, los sistemas adhesivos autoacondicionantes

Tabla I. Adhesivos Convencionales.

\begin{tabular}{llll}
\hline NOMBRE & MARCA COMERCIAL & SOLVENTE & MONÓMEROS \\
\hline Optibond 5 & Kerr & Etanol & HEMA/GDPM/TEGDMA/UDMA \\
XP Bond & Dentsply & Butanol & PENTA/TEGDMA/UDMA/TBC \\
Adper Single Bond 2 & 3M ESPE & Etanol-Agua & BisGMA/HEMA \\
Prime \& Bond NT & Dentsply & Acetona-Etanol & DEGMA/PENTA/BisGMA/UDMA \\
Solobond M & VOCO & Acetona & BisGMA/HEMA/BHT \\
One Coat Bond SL & Coltène/Whaledent & Agua & HEMA/*HEPMA/UDMA \\
\hline
\end{tabular}

*HEPMA no se pudo identificar. 
Tabla II. Adhesivos Autoacondicionantes.

\begin{tabular}{llll}
\hline Nombre & Marca Comercial & Solvente & Monómeros \\
\hline AdheSE & Ivoclar/Vivadent & Agua & Bis-acrilamida/ Bis-meta e \\
Futurabond DC & VOCO & Etanol & BisGMA/HEMA/TMPTMA/BHT DABA \\
Adper Silorano & 3M ESPE & Agua-Etanol & BisGMA/HEMA/Silorano \\
Go! & SDI & Agua-Acetona & BisGMA/HEMA \\
\hline
\end{tabular}

de uso odontológico presentan una mayor degradación que los sistemas adhesivos convencionales al estudiar su comportamiento térmico, bajo diferentes condiciones ambientales.

Los cambios observados en la curva pérdida de masa v/s temperatura de los termogramas, se asociaron a diferentes procesos de pérdida de masa por procesos de degradación térmica del material. Fue difícil establecer las temperaturas en las cuales ocurrían estos procesos, por este motivo se obtuvo la primera derivada de la curva TGA que se presentó como línea segmentada. Los cambios en la primera derivada fueron visualizados como peaks, atribuidos a la temperatura en la cual sucedieron estos procesos degradativos.

Desde los termogramas se midió la pérdida de masa y cantidad de masa residual, como también, la cantidad de masa que se descompone en cada etapa de des-

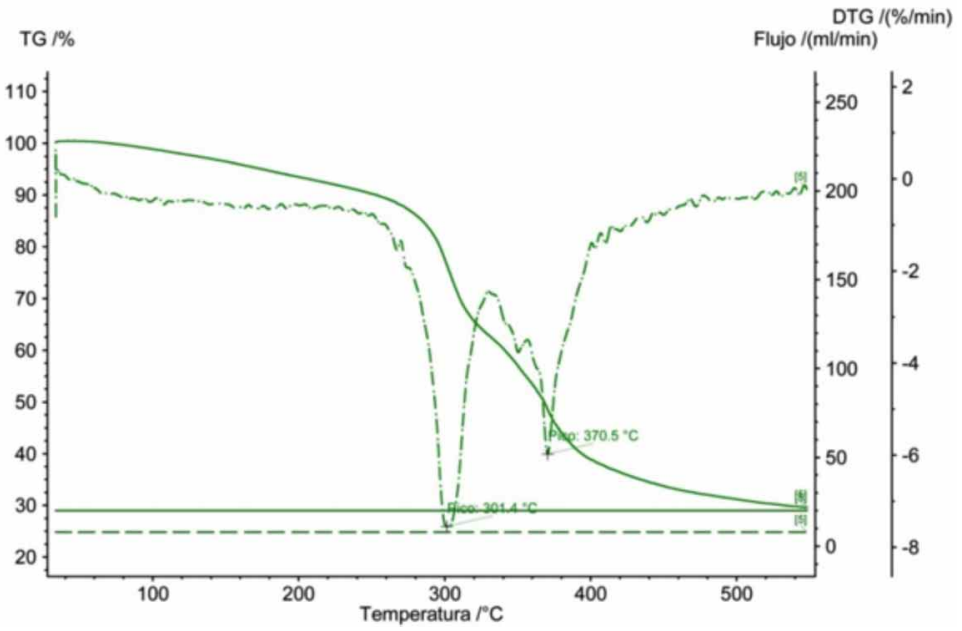

Fig.1. Termogravimetría de Futurabond DC. Es posible identificar tres procesos de descomposición o degradación térmica del material. La cantidad de material residual asignado a carga o componentes inorgánicos es $29.61 \%$.Desde la curva segmentada se puede observar la temperatura a las cuales suceden estos procesos degradativos. El primero a $301.4^{\circ} \mathrm{C}$, el segundo a $350{ }^{\circ} \mathrm{C}$ y el tercero a $370.5^{\circ} \mathrm{C}$. En el primer proceso degradativo se pierde un $23.2 \%$ de masa. Bajo los $300{ }^{\circ} \mathrm{C}$ se pierde $10 \%$ de masa. A $350{ }^{\circ} \mathrm{C}$ se pierde $20 \%$ de masa. A $370.5^{\circ} \mathrm{C}$ se pierden $51.8 \%$ de masa.

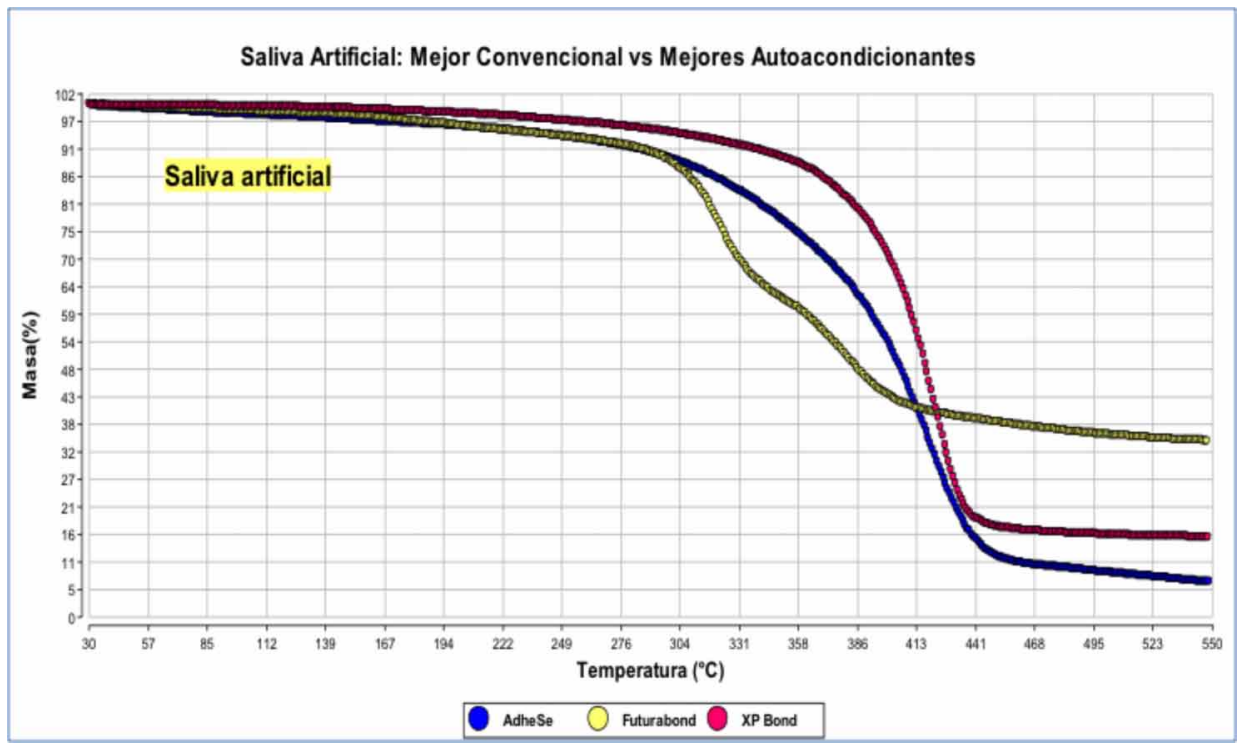

Fig. 2. Comparación del sistema adhesivo convencional y el sistema adhesivo autoacondicionante que presenta menor degradación en saliva artificial. Desde el gráfico se puede inferir que el sistema adhesivo autoacondicionante con mejor estabilidad térmica en ambiente saliva artificial es AdheSe, el que comienza su degradación a $418,6^{\circ} \mathrm{C}$. El sistema convencional más estable es $X P$ Bond, que se degrada a 422,2 ${ }^{\circ} \mathrm{C}$. Se destaca así, que el sistema adhesivo convencional o de grabado independiente es el más estable en este ambiente. 
composición. Todos los adhesivos presentaron un patrón TGA que permitió establecer la dinámica de degradación, siendo diferentes de acuerdo al sistema adhesivo. Similarmente, se pudo establecer que los sistemas adhesivos presentaron diferente cantidad de masa residual o carga inorgánica que no se descompone en el rango de temperatura del proceso degradativo.

En general, todos los sistemas adhesivos presentaron varios peaks, lo que significó que tenían varios procesos degradativos. La cantidad de peaks y los valores de temperatura para cada uno de ellos, indicó la estabilidad del material o eficiencia de la polimerización de ellos. Se identificaron los sistemas adhesivos comerciales que presentan menor pérdida de masa. Si existía solo un proceso o etapa de des-

Tabla III. Sistemas adhesivos más estables al efecto de la temperatura, y por lo tanto con menor degradación, en cada uno de los ambientes o medios.

\begin{tabular}{lll}
\hline \multicolumn{1}{c}{ AMBIENTES } & & $\mathbf{1}^{\circ}$ Derivada ${ }^{\circ} \mathrm{C}$ \\
\hline Humedad relativa & AdheSe & $414,8{ }^{\circ} \mathrm{C}$ \\
(grupo control) & Adper Silorano & $405,7{ }^{\circ} \mathrm{C}$ \\
& Adper Single Bond 2 & $423,2^{\circ} \mathrm{C}$ \\
& Optibond S & $422,8{ }^{\circ} \mathrm{C}$ \\
$100 \%$ Agua & Adper Silorano & $405,2{ }^{\circ} \mathrm{C}$ \\
& AdheSe & $404,4{ }^{\circ} \mathrm{C}$ \\
& Optibond S & $422{ }^{\circ} \mathrm{C}$ \\
$\mathrm{pH} 3$ & Adper Single Bond 2 & $420,3{ }^{\circ} \mathrm{C}$ \\
& AdheSe & $416,7{ }^{\circ} \mathrm{C}$ \\
& Adper Silorano & $408,1{ }^{\circ} \mathrm{C}$ \\
Saliva artificial & Optibond S & $426{ }^{\circ} \mathrm{C}$ \\
& XP Bond & $425,2{ }^{\circ} \mathrm{C}$ \\
& AdheSe & $418,6{ }^{\circ} \mathrm{C}$ \\
& Go! & $412,3{ }^{\circ} \mathrm{C}$ \\
& Optibond S & $426,2{ }^{\circ} \mathrm{C}$ \\
& Prime \& Bond NT & $424,1{ }^{\circ} \mathrm{C}$ \\
\hline
\end{tabular}

composición, la curva integral presentó un único proceso de pérdida de masa, y en consecuencia, la primera derivada mostró un único peak. En general se considera, que los menores porcentaje de pérdidas de de masa corresponden a los sistemas adhesivos más estables con menor pérdida de masa y una relación de degradación menor. Todos los sistemas adhesivos se encontraron altamente polimerizados por tener en su composición compuestos de elevado peso molecular.

Además de las fórmulas de los monómeros que componen los polímeros, se identificaron los monómeros que dan origen a los polímeros e inhibidores de la polimerización BHT y TBC. Fue posible determinar los compuestos químicos que forman los sistemas adhesivos poliméricos, de acuerdo a la información entregada por los fabricantes. En todos está presente la banda a $1725 \mathrm{~cm}^{-1}$ correspondiente a la vibración de los enlaces $\mathrm{CR}_{1} \mathrm{CR}_{2}=\mathrm{CH}_{2}$ de compuestos vinílicos disustituidos, indicando que la polimerización no ocurre totalmente (Fig. 3).

\section{DISCUSIÓN}

Son múltiples las preguntas que se desea responder al realizar este trabajo de investigación, en el ámbito de la amplia y a veces indiscriminada utilización de sistemas adhesivos en la odontología contemporánea. A la luz de los resultados obtenidos, se da respuesta a los objetivos planteados, al cuantificar la alteración que sufren los sistemas adhesivos poliméricos analizados, por cambios térmicos en diversos ambientes y su correlación con el estudio por espectroscopía infrarrojo FT-IR.

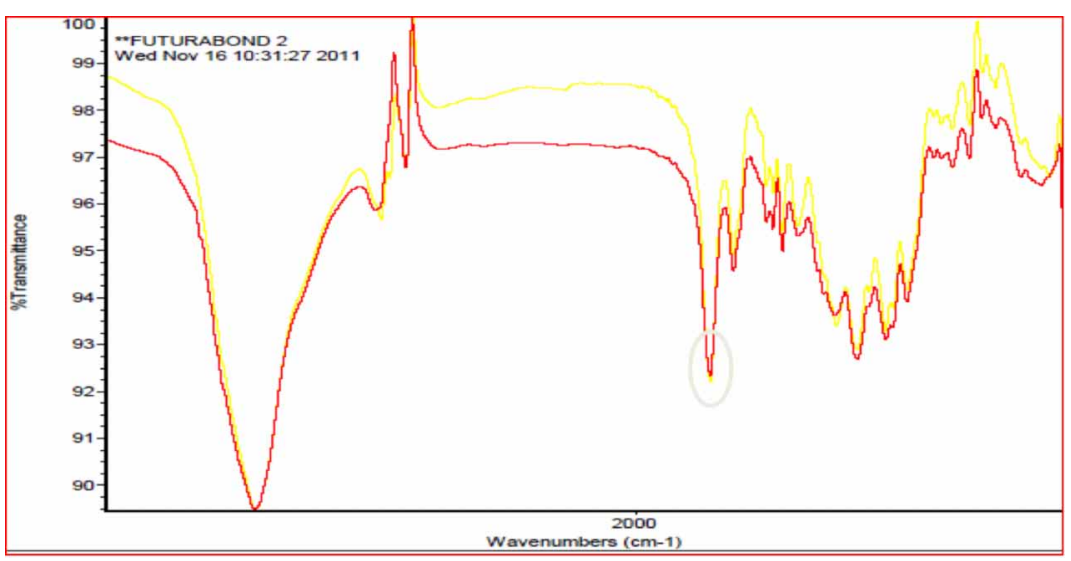

Fig. 3. Termogravimetría de Futurabond DC. En todos los casos está presente la banda a alrededor de $1725 \mathrm{~cm}^{-1}$ correspondiente a la vibración de los enlaces $\mathrm{CR}_{1} \mathrm{CR}_{2}=\mathrm{CH}_{2}$ de compuestos vinílicos disustituidos, lo que indica que las reacciones de polimerización no ocurren totalmente. 
El examen por FT-IR indica las bandas de los grupos funcionales de cada compuesto. Este análisis presenta los grupos funcionales orgánicos más importantes de los distintos adhesivos, destacando la cantidad de grupos $\mathrm{OH}$, y si está correlacionado con el comportamiento térmico. Sólo se pudo saber con cierta certeza el tipo de monómero, pero no la estructura química completa del monómero específico. Se comprobó que los sistemas adhesivos están formados por los monómeros que los fabricantes declaran en sus instructivos (Fig. 4).

La pérdida de masa por la degradación, producto de la hidrólisis de los sistemas adhesivos en boca, se produce por las condiciones limitantes y adversas del medio bucal, en donde se obtienen los polímeros a partir de mezclas complejas de monómeros resinosos, diluyentes y solventes. En cambio, su fabricación, se realiza en condiciones ideales de deshidratación, presión, temperatura, tiempo, etc. pronosticando de esta manera una durabilidad prolongada, situación que no ocurre en la cavidad bucal (Carvahlo et al., 2003).

Todos los sistemas adhesivos del mercado obtienen buenos resultados inmediatos. La complejidad se presenta en el desgaste en el tiempo de la interface adhesiva. Los sistemas adhesivos de menos pasos son más sencillos de usar pero sacrifican la calidad y duración de la adhesión. Se recomienda emplear un sellador superficial hidrófugo, extender los tiempos de polimerización, usar inhibidores de las MMPs, mejorar la impregnación del sistema adhesivo aumentando los tiempos de aplicación y frotarlo enérgicamente.

La adhesión dentaria abarca todo el sistema de unión entre diversos materiales dentales y los distintos sustratos dentarios, ya sean esmalte o dentina. Cada sistema adhesivo tiene sus ventajas y desventajas dependiendo del sustrato dentario. La simplificación de la técnica es lo que se busca actualmente, pero el estándar de oro de la adhesión sigue siendo a través de múltiples pasos.

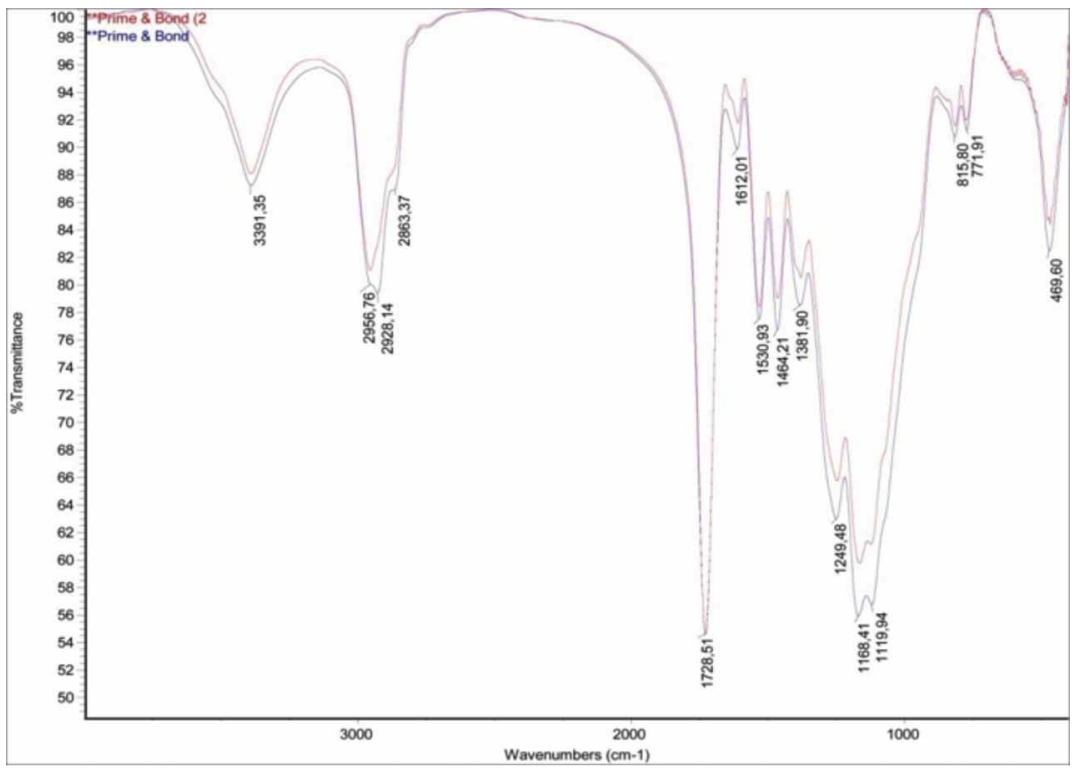

NASS, K. L. \& CARPENTIERI, A. R. Correlation between thermal behavior and composition of adhesives systems. Int. J. Odontostomat., 10(1):53-60, 2017.

ABSTRACT: An in vitro study of eleven adhesive systems was carried out to establish if clinical work conditions could improve their efficiency. It has been observed that self-etch adhesive systems have less thermal stability than conventional ones. As a consequence, early replacement of unsatisfactory aesthetic restorations is needed. The objectives of this work were to determine the thermal behavior of eleven polymeric adhesive systems under different rates of temperatures and a variety of conditions such as $100 \%$ humidity, presence of acidulated water $(\mathrm{pH} 3)$, environmental relative humidity and artificial saliva. Another aim was to establish if these factors are correlated with the adhesive systems composition. For each type of adhesive system, samples were prepared in small aluminum caps and polymerized with a LED lamp. All samples were analyzed with the Fourier-Transform Infrared Spectroscopy (FT-IR) method, which assigned absorption bands to organic functional groups of each compound corresponding to their chemical type. Subsequently, a thermogravimetric analysis was performed in a range temperature from room temperature to $500{ }^{\circ} \mathrm{C}$ in order to establish thermal behavior in an inert environment and after staying in acidulated water, $100 \%$ humidity, environmental humidity and ar-

Fig. 4. Caracterización por FT-IR de Prime \& Bond NT (Dentsply) El polímero obtenido por polimerización radical del adhesivo monomérico nombrado como Prime \& Bond presenta las siguientes bandas de vibración de tensión: $v-\mathrm{c}=\mathrm{O}$ a $1727 \mathrm{~cm}^{-1} ; \mathrm{n} 2957$ y 1464 de grupos $-\mathrm{CH}_{3}$ $y-\mathrm{CH}_{2}-$, los grupos fosfatos $\mathrm{P}=\mathrm{O}$ se presentan a 1249 y $1168 \mathrm{~cm}^{-1}$. Los adhesivos monoméricos pueden estar formados por Bis-GMA, UDMA, DEGMA (dietilenglicol dimetacrilato) y PENTA (dipentaeritrol-penta-acrilato-ácido-estermono fosforado). 
tificial saliva. Thermograms were obtained to collect data about decomposition temperatures and loss of mass percentages. The FT-IR study results were correlated with the adhesive systems thermal behavior. Thermogram images showed loss of mass, polymerization efficiency and residual mass amount. The different degradation dynamics were analyzed according to curve designs and baseline changes. Both groups of adhesive systems revealed high polymerization degrees. Nevertheless, the effect produced by the conditions in which they were subjected depends on the type of adhesive. Conventional adhesives, in contrast to self-etch adhesives, degraded in a minor extension as a result of their higher thermal stability.

KEY WORDS: adhesive systems, thermal stability, hydrolytic degradation.

\section{REFERENCIAS BIBLIOGRÁFICAS}

Breschi, L.; Mazzoni, A.; Ruggeri, A.; Cadenaro, M.; Di Lenarda, R. \& De Stefano Dorigo, E. Dental adhesion review: aging and stability of the bonded interface. Dent. Mater., 24(1):90-101, 2008.

Carvahlo, R. M.; Carrillo, M. R. O.; Pereira, L. C. G.; García, F. C. P. \& Marquezini, L. Durabilidad de la Unión Resina-Diente. Una Dimensión Olvidada. Curitiba, Ed. Maio, 2003. pp.441-54.

De Munck, J.; Van Landuyt, K.; Peumans, M.; Poitevin, A.; Lambrechts, P.; Braem, M. \& Van Meerbeek, B. A critical review of the durability of adhesion to tooth tissue: methods and results. J. Dent. Res., 84(2):118-32, 2005.

Donassolo, T. A.; Osinaga, P. W. R.; Demarco, F. F. \& Piva, E. Mass variation of dentin adhesives system stored in different solutions. Oral Sci., 1(1):29-34, 2005.

Eminkahyagil, N.; Gokalp, S.; Korkmaz, Y.; Baseren, M. \& Karabulut, E. Sealant and composite bond strength to enamel with antibacterial/self-etching adhesives. Int. J. Paediatr. Dent., 15(4):274-81, 2005.

Fuks, A. B.; Eidelman, E. \& Lewinstein, I. Shear strength of sealants placed with non-rinse conditioning compared to a conventional acid etch-rinse technique. A. S. D. C. J. Dent. Child., 69(3):23942, 2002.

Gamborgi, G. P.; Loguercio, A. D. \& Reis, A. Influence of enamel border and regional variability on durability of resin-dentin bonds. J. Dent., 35(5):371-6, 2007.

Hannig, M.; Gräfe, A.; Atalay, S. \& Bott, B. Microleakage and SEM evaluation of fissure sealants placed by use of self-etching priming agents. J. Dent., 32(1):75-81, 2004.

Hashimoto, M.; Ohno, H.; Kaga, M.; Endo, K.; Sano, H. \& Oguchi, $\mathrm{H}$. In vivo degradation of resin-dentin bonds in humans over 1 to 3 years. J. Dent. Res., 79(6):1385-91, 2000.

Kostoryz, E. L.; Dharmala, K.; Ye, Q.; Wang, Y.; Huber, J.; Park, J. G.; Snider, G.; Katz, J. L. \& Spencer, P. Enzymatic biodegradation of HEMA/bisGMA adhesives formulated with different water content. J. Biomed. Mater Res. B Appl. Biomater., 88(2):394401, 2009

Leinfelder, K. F. Do restorations made of amalgam outlast those made of resin-based composite? J. Am. Dent. Assoc., 131(8):1186-7, 2000.

Peumans, M.; Kanumilli, P.; De Munck, J.; Van Landuyt, K.; Lambrechts, P. \& Van Meerbeek, B. Clinical effectiveness of contemporary adhesives: a systematic review of current clinical trials. Dent. Mater., 21(9):864-81, 2005.
Perdigão, J.; Fundingsland, J. W.; Duarte, S. Jr. \& Lopes, M. Microtensile adhesion of sealants to intact enamel. Int. J. Paediatr. Dent., 15(5):342-8, 2005.

Peutzfeldt, A. \& Nielsen, L. A. Bond strength of a sealant to primary and permanent enamel: phosphoric acid versus self-etching adhesive. Pediatr. Dent., 26(3):240-4, 2004.

Ritter, A. V.; Swift, E. J. Jr.; Heymann, H. O.; Sturdevant, J. R. \& Wilder, A. D. Jr. An eight-year clinical evaluation of filled and unfilled one-bottle dental adhesives. J. Am. Dent. Assoc., 140(1):28-37, 2009.

Rodríguez G., D. R. \& Pereira S., N. A. Evolución y tendencias actuales en resinas compuestas Acta Odontol. Venez., 46(3), 2008. Disponible en: www.actaodontologica.com/ediciones/2008/3/ evolucion_tendencias_resinas_compuestas.asp

Rouessac, F. \& Rouessac, A. Chemical Analysis. Modern Instrumentation Methods and Techniques. New York, Wiley, 2000. pp.161-87.

Sano, H.; Yoshikawa, T.; Pereira, P. N.; Kanemura, N.; Morigami, M.; Tagami, J. \& Pashley, D. H. Long-term durability of dentin bonds made with a self-etching primer, in vivo. J. Dent. Res., 78(4):906-11, 1999.

Spencer, P.; Wang, Y. \& Bohaty, B. Interfacial chemistry of moistureaged class II composite restorations. J. Biomed. Mater. Res. B Appl. Biomater., 77(2):234-40, 2006.

Takahashi, M.; Nakajima, M.; Hosaka, K.; Ikeda, M.; Foxton, R. M. \& Tagami, J. Long-term evaluation of water sorption and ultimate tensile strength of HEMA-containing/-free one-step self-etch adhesives. J. Dent., 39(7):506-512, 2011.

Tay, F. R.; Gwinnett, A. J. \& Wei, S. H. The overwet phenomenon: an optical, micromorphological study of surface moisture in the acid-conditioned, resin-dentin interface. Am. J. Dent., 9(1):43-8, 1996.

Dirección para correspondencia:

Lilian Nass Kunstmann

Facultad de Odontología

Universidad de Concepción

Concepción

CHILE

Email: Inass@udec.cl

Recibido : 24-10-2016

Aceptado: 26-12-2016 